\title{
Powers of the Vandermonde determinant, Schur functions, and the dimension game
}

\author{
Cristina Ballantine ${ }^{1}$ \\ ${ }^{1}$ Department of Mathematics and Computer Science, College of the Holy Cross, Worcester, MA, USA
}

\begin{abstract}
Since every even power of the Vandermonde determinant is a symmetric polynomial, we want to understand its decomposition in terms of the basis of Schur functions. We investigate several combinatorial properties of the coefficients in the decomposition. In particular, I will give a recursive approach for computing the coefficient of the Schur function $s_{\mu}$ in the decomposition of an even power of the Vandermonde determinant in $n+1$ variables in terms of the coefficient of the Schur function $s_{\lambda}$ in the decomposition of the same even power of the Vandermonde determinant in $n$ variables if the Young diagram of $\mu$ is obtained from the Young diagram of $\lambda$ by adding a tetris type shape to the top or to the left.

Résumé. Comme toute puissance paire du déterminant de Vandermonde est un polynôme symétrique, nous voulons comprendre sa décomposition dans la base des fonctions de Schur. Nous allons étudier plusieurs propriétés combinatoires des coefficients de la décomposition. En particulier, nous allons donner une approche récursive pour le calcul du coefficient de la fonction de Schur $s_{\mu}$ dans la décomposition d'une puissance paire du déterminant de Vandermonde en $n+1$ variables, en fonction du coefficient de la fonction de Schur $s_{\lambda}$ dans la décomposition de la même puissance paire du déterminant de Vandermonde en $n$ variables, lorsque le diagramme de Young de $\mu$ est obtenu à partir du diagramme de Young de $\lambda$ par l'addition d'une forme de type tetris vers le haut ou vers la gauche.
\end{abstract}

Keywords: Schur functions, Vandermonde determinant, Young diagrams, symmetric functions, quantum Hall effect

\section{Introduction}

Because Vandermonde determinants are so prevalent in mathematics I will not attempt to list here their many applications. In the theory of formal power series they are best known for the part they play in the classical definition of Schur functions. Since each even power of the Vandermonde determinant is a symmetric function, it is natural to ask for its decomposition in terms of the basis for the ring of symmetric functions given by Schur functions. This decomposition has been studied extensively (see [2], [3], [7], and the references therein) in connection with its usefulness in the understanding of the (fractional) quantum Hall effect. In particular, the coefficients in the decomposition correspond precisely to the coefficients in the decomposition of the Laughlin wave function as a linear combination of (normalized) Slater determinantal wave functions. The calculation of the coefficients in the decomposition becomes computationally expensive as the size of the determinant increases. Several algorithms for the expansion of the square of the Vandermonde determinant in terms of Schur functions are available (see, for example [7]). However, a combinatorial interpretation for the coefficient of a given Schur function is still unknown. Recently, 
Boussicault, Luque and Tollu [1] provided a purely numerical algorithm for computing the coefficient of a given Schur function in the decomposition without computing the other coefficients. The algorithm uses hyperdeterminants and their Laplace expansion. It was used by the authors to compute coefficients in the decomposition of even powers of Vandermonde determinants of size up to 11. For determinants of large size, the algorithm becomes computationally too expensive for practical purposes. In this article we present recursive combinatorial properties of some of the coefficients on the decomposition.

\subsection{Statement of results}

We denote by $a_{\delta_{n}}$ the Vandermonde determinant $a_{\delta_{n}}=\operatorname{det}\left(x_{i}^{n-j}\right)_{i, j=1}^{n}=\prod_{1 \leq i<j \leq n}\left(x_{i}-x_{j}\right)$. For fixed positive integers $n$ and $k$, suppose $\lambda$ is a partition of $k n(n-1)$ and $\mu$ is a partition of $k n(n+1)$. We prove the following results relating $\left\langle a_{\delta_{n+1}}^{2 k}, s_{\mu}\right\rangle$ and $\left\langle a_{\delta_{n}}^{2 k}, s_{\lambda}\right\rangle$ when the diagram of $\mu$ is obtained by adding a certain configuration of boxes, called a tetris type shape, to the top or to the left of the diagram of $\lambda$.

1. (Theorem 4.1) If the Young diagram of $\mu$ is obtained by adding the tetris type shape of size $2 k n$

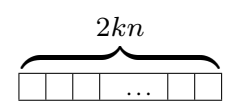

to the top of the Young diagram of $\lambda$, then $\left\langle a_{\delta_{n+1}}^{2 k}, s_{\mu}\right\rangle=\left\langle a_{\delta_{n}}^{2 k}, s_{\lambda}\right\rangle$.

2. (Corollary 4.3 ) If the Young diagram of $\mu$ is obtained by adding the tetris type shape of size $2 k n$

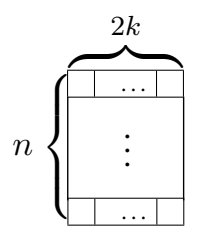

to the left of the Young diagram of $\lambda$, then $\left\langle a_{\delta_{n+1}}^{2 k}, s_{\mu}\right\rangle=\left\langle a_{\delta_{n}}^{2 k}, s_{\lambda}\right\rangle$.

For the remaining results let $k=1$.

3. (Theorem 4.6 If the Young diagram of $\mu$ is obtained by adding the tetris type shape of size $2 n$

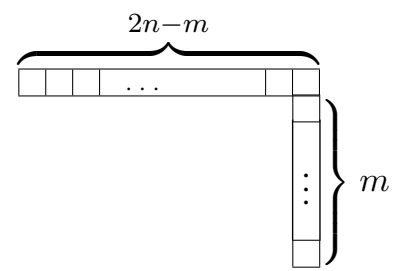

to the top of the Young diagram of $\lambda$, then $\left\langle a_{\delta_{n+1}}^{2}, s_{\mu}\right\rangle=(-1)^{m}(2 m+1)\left\langle a_{\delta_{n}}^{2}, s_{\lambda}\right\rangle$.

4. (Conjecture 4.8) If the Young diagram of $\mu$ is obtained by adding the tetris type shape of size $2 n$

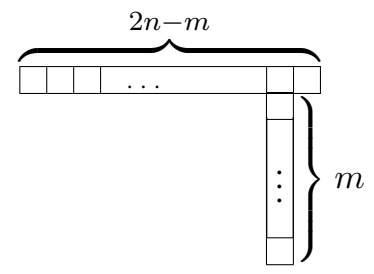


to the top of theYoung diagram of $\lambda$, then $\left\langle a_{\delta_{n+1}}^{2}, s_{\mu}\right\rangle=(-1)^{m}(m+1)\left\langle a_{\delta_{n}}^{2}, s_{\lambda}\right\rangle$.

5. (Theorem 4.12 If the Young diagram of $\mu$ is obtained by adding the tetris type shape of size $2 n$

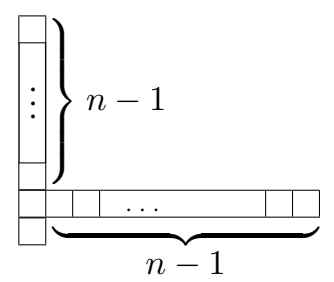

to the left of the Young diagram of $\lambda$, then $\left\langle a_{\delta_{n+1}}^{2}, s_{\mu}\right\rangle=(-1)^{n} 3 n\left\langle a_{\delta_{n}}^{2}, s_{\lambda}\right\rangle$.

We also prove several corollaries of the results above.

\section{Notation and basic facts}

We first introduce some notation and basic facts about Vandermonde determinants related to this problem. For details on partitions and Schur functions we refer the reader to [6, Chapter 7].

Let $n$ be a non-negative integer. A partition of $n$ is a weakly decreasing sequence of non-negative integers, $\lambda:=\left(\lambda_{1}, \lambda_{2}, \cdots, \lambda_{\ell}\right)$, such that $|\lambda|:=\sum \lambda_{i}=n$. We write $\lambda \vdash n$ to mean $\lambda$ is a partition of $n$. The nonzero integers $\lambda_{i}$ are called the parts of $\lambda$. We identify a partition with its Young diagram, i.e. the array of left-justified squares (boxes) with $\lambda_{1}$ boxes in the first row, $\lambda_{2}$ boxes in the second row, and so on. The rows are arranged in matrix form from top to bottom. By the box in position $(i, j)$ we mean the box in the $i$-th row and $j$-th column of $\lambda$. The length of $\lambda, \ell(\lambda)$, is the number of rows in the Young diagram or the number of non-zero parts of $\lambda$. For example,

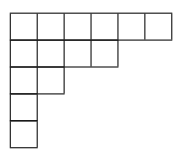

is the Young diagram for $\lambda=(6,4,2,1,1)$, with $\ell(\lambda)=5$ and $|\lambda|=14$.

We write $\lambda=\left\langle 1^{m_{1}}, 2^{m_{2}} \ldots\right\rangle$ to mean that $\lambda$ has $m_{i}$ parts equal to $i$.

Given a weak composition $\alpha=\left(\alpha_{1}, \alpha_{2}, \ldots \alpha_{n}\right)$ of length $n$, we write $x^{\alpha}$ for $x_{1}^{\alpha_{1}} x_{2}^{\alpha_{2}} \ldots x_{n}^{\alpha_{n}}$. If $\lambda=\left(\lambda_{1}, \lambda_{2}, \ldots, \lambda_{n}\right)$ is a partition of length at most $n$ and $\delta=\delta_{n}=(n-1, n-2, \ldots, 2,1,0)$, then the skew symmetric function $a_{\lambda+\delta}$ is defined as

$$
a_{\lambda+\delta}=\operatorname{det}\left(x_{i}^{\lambda_{j}+n-j}\right)_{i, j=1}^{n}
$$

If $\lambda=\emptyset$,

$$
a_{\delta}=\operatorname{det}\left(x_{i}^{n-j}\right)_{i, j=1}^{n}=\prod_{1 \leq i<j \leq n}\left(x_{i}-x_{j}\right)
$$

is the Vandermonde determinant. We have [6, Theorem 7.15.1]

$$
a_{\lambda+\delta} / a_{\delta}=s_{\lambda}\left(x_{1}, \ldots, x_{n}\right)
$$


where $s_{\lambda}\left(x_{1}, \ldots, x_{n}\right)$ is the Schur function of shape $\lambda$ in variables $x_{1}, \ldots, x_{n}$. Moreover, for any homogeneous symmetric function $f$ of degree $n$, we have [6, Corollary 7.15.2]

$$
\left\langle f, s_{\lambda}\right\rangle_{n}=\left[x^{\lambda+\delta}\right] a_{\delta} f
$$

i.e., the coefficient of $x^{\lambda+\delta}$ in $a_{\delta} f$. In particular, if $f=a_{\delta}^{2 k}$, then

$$
\left\langle a_{\delta}^{2 k}, s_{\lambda}\right\rangle=\left[x^{\lambda+\delta}\right] a_{\delta}^{2 k+1} .
$$

We will often write $c_{\lambda}$ for $\left\langle a_{\delta_{n}}^{2 k}, s_{\lambda}\right\rangle$.

The goal of this work is to investigate several combinatorial properties of the numbers (1).

We note that $|\delta|=n(n-1) / 2$ and $a_{\delta}$ is a homogeneous polynomial of degree $n(n-1) / 2$. If $\left\langle a_{\delta}^{2 k}, s_{\lambda}\right\rangle \neq$ 0 , then $|\lambda|=k n(n-1), n-1 \leq \ell(\lambda) \leq n, k(n-1) \leq \lambda_{1} \leq k n(n-1)$ and $\lambda_{n} \leq k(n-1)$. Moreover, if $\lambda_{n}=k(n-1)$, then $\lambda=(k(n-1))^{n}$.

Whenever it is necessary to emphasize the dimension, we write $\delta_{n}$ for $\delta$ and $a_{\delta_{n}}$ for $a_{\delta}$.

By $\bar{a}_{\delta_{n}}$ we mean $a_{\delta_{n}}$ with $x_{i}$ replaced by $x_{i+1}$ for each $i=1,2, \ldots n$. Thus,

$$
\bar{a}_{\delta_{n}}=\prod_{2 \leq i<j \leq n+1}\left(x_{i}-x_{j}\right)
$$

Given a weak composition $\alpha=\left(\alpha_{1}, \alpha_{2}, \ldots, \alpha_{n}\right)$ of $n$ of length $n$, we denote by $c_{\alpha}$ the coefficient of $x^{\alpha}$ in $a_{\delta_{n}}^{2 k+1}$. If $\xi$ is a permutation of $\{1,2, \ldots, n\}$, and $\xi(\alpha)$ is the weak composition $\left(\alpha_{\xi(1)}, \alpha_{\xi(2)}, \ldots, \alpha_{\xi(n)}\right)$, then $c_{\alpha}=\operatorname{sgn}(\xi) c_{\xi(\alpha)}$.

To simplify the notation, we write $\lambda=\left(\lambda_{1}, \lambda_{2}, \ldots, \lambda_{n}\right)$ for a partition with $\ell(\lambda) \leq n$ by setting $\lambda_{j}=0$ if $j>\ell(\lambda)$.

\section{The box-complement of a partition}

Definition: Let $\lambda=\left(\lambda_{1}, \lambda_{2}, \ldots, \lambda_{n-1}, \lambda_{n}\right)$ be a partition of $k n(n-1)$ with $\ell(\lambda) \leq n$. The boxcomplement of $\lambda$ is the partition of $k n(n-1)$ given by

$$
\lambda_{b c}:=\left(2 k(n-1)-\lambda_{n}, 2 k(n-1)-\lambda_{n-1}, \ldots, 2 k(n-1)-\lambda_{2}, 2 k(n-1)-\lambda_{1}\right) .
$$

Thus, $\lambda_{b c}$ is obtained from $\lambda$ in the following way. Place the Young diagram of $\lambda$ in the upper left corner of a box with $n$ rows each of length $2 k(n-1)$. If we remove the Young diagram of $\lambda$ and rotate the remaining shape by $180^{\circ}$, we obtain the Young diagram of $\lambda_{b c}$.

Example: Let $k=1, n=4$ and $\lambda=(5,3,2,2)$. Then $\lambda_{b c}=(4,4,3,2)$. The Young diagram of $\lambda$ is shown on the left of the $4 \times 6$ box. The remaining squares of the box are marked with $X$. They form the diagram of $\lambda_{b c}$ rotated by $180^{\circ}$.

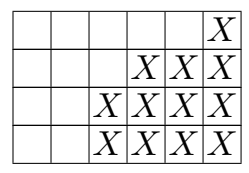


Lemma 3.1 (Box-complement lemma) With the notation above we have

$$
\left\langle a_{\delta}^{2 k}, s_{\lambda}\right\rangle=\left\langle a_{\delta}^{2 k}, s_{\lambda_{b c}}\right\rangle .
$$

For a proof in the case $k=1$, see [2, Section 6] where the box-complement partition is referred to as the reversed partition. We prove the lemma for general $k$ by elementary means, using induction on $n$. In [2], Dunne also explains the physical meaning of the box-complement lemma.

\section{The dimension game}

The goal of this section is to establish a relationship between $\left\langle a_{\delta_{n+1}}^{2 k}, s_{\mu}\right\rangle$ and $\left\langle a_{\delta_{n}}^{2 k}, s_{\lambda}\right\rangle$ when there is a relationship between $\lambda \vdash k n(n-1)$ and $\mu \vdash k n(n+1)$ of the type described in the introduction.

Theorem 4.1 If $\lambda=\left(\lambda_{1}, \lambda_{2}, \ldots, \lambda_{n}\right)$ is a partition of $k n(n-1)$ with $\ell(\lambda) \leq n$ and $\mu$ is the partition of $k n(n+1)$ given by $\mu=\left(2 k n, \lambda_{1}, \lambda_{2}, \ldots, \lambda_{n}\right)$, then

$$
\left\langle a_{\delta_{n+1}}^{2 k}, s_{\mu}\right\rangle=\left\langle a_{\delta_{n}}^{2 k}, s_{\lambda}\right\rangle
$$

Thus, adding the tetris type shape

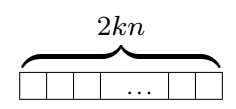

to the top of the diagram for $\lambda$ does not change the coefficient.

Proof: The proof follows by induction from

$$
a_{\delta_{n+1}}^{2 k+1}=\prod_{i=2}^{n+1}\left(x_{1}-x_{i}\right)^{2 k+1} \cdot \bar{a}_{\delta_{n}}^{2 k+1} .
$$

Remark: The theorem is also true if $\lambda$ is just a weak composition of $k n(n-1)$ (with no more than one part equal to 0 ).

Note: The result of the theorem for $k=1$ is also noted in (23a) of [7].

Corollary 4.2 If $\lambda=(2 k(n-1), 2 k(n-2), \ldots, 4 k, 2 k, 0)=2 k \delta_{n}$, then $\left\langle a_{\delta_{n}}^{2 k}, s_{\lambda}\right\rangle=1$.

Using Theorem 4.1 and Lemma 3.1 , we obtain the following corollary.

Corollary 4.3 If $\lambda=\left(\lambda_{1}, \lambda_{2}, \ldots, \lambda_{n}\right)$ is a partition of $k n(n-1)$ with $\ell(\lambda) \leq n$, and $\mu$ is the partition of $k n(n+1)$ given by $\mu=\lambda+\left\langle(2 k)^{n}\right\rangle=\left(\lambda_{1}+2 k, \lambda_{2}+2 k, \ldots, \lambda_{n}+2 k\right)$, then

$$
\left\langle a_{\delta_{n+1}}^{2 k}, s_{\mu}\right\rangle=\left\langle a_{\delta_{n}}^{2 k}, s_{\lambda}\right\rangle
$$

Thus adding the tetris type shape

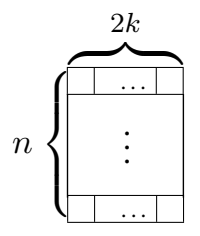


to the left of the diagram of $\lambda$ does not change the coefficient.

Note: For $k=1$ this is (23b) of [7].

For the remainder of the article we set $k=1$.

Lemma 4.4 Suppose $\lambda \vdash n(n-1)$ with $n-1 \leq \ell(\lambda) \leq n$ and $\left\langle a_{\delta_{n}}^{2}, s_{\lambda}\right\rangle \neq 0$. If $\lambda_{n}=\lambda_{n-1}=\ldots=$ $\lambda_{n-i}=s$, then $i \leq s$, i.e., the maximum number of rows of size $s$ at the bottom of the diagram is $s+1$.

Proof: We examine the coefficient of $x^{\lambda}+\delta_{n}$ in $a_{\delta_{n}}^{3}$. Suppose $i=s+1$. Then

$$
x^{\lambda+\delta_{n}}=\left(x_{1}^{\lambda_{1}+n-1} \cdots x_{n-s}^{\lambda_{n-s}+s}\right) \cdot M_{s},
$$

where the monomial $M_{s}=x_{n-s-1}^{2 s+1} \cdots x_{n-2}^{s+2} x_{n-1}^{s+1} x_{n}^{s}$ has degree $\frac{(3 s+1)(s+2)}{2}$.

On the other hand,

$$
\prod_{j=n-s}^{n}\left(x_{n-s-1}-x_{j}\right)^{3} \cdot \prod_{j=n-s+1}^{n}\left(x_{n-s}-x_{j}\right)^{3} \cdots \prod_{j=n-1}^{n}\left(x_{n-2}-x_{j}\right)^{3} \cdot\left(x_{n-1}-x_{n}\right)^{3}
$$

contributes powers of $x_{n-s-1}, x_{n-s}, \ldots, x_{n}$ to all monomials in $a_{\delta_{n}}^{3}$. However, each monomial in the product 13 has degree $\frac{(3 s+3)(s+2)}{2}$. Therefore, $i \leq s$.

Reformulating the previous lemma in terms of the box-complement of the partition $\lambda$, we obtain the following corollary.

Corollary 4.5 Suppose $\lambda \vdash n(n-1)$ with $n-1 \leq \ell(\lambda) \leq n$ and $\left\langle a_{\delta_{n}}^{2}, s_{\lambda}\right\rangle \neq 0$. If $\lambda_{1}=\lambda_{2}=\ldots=$ $\lambda_{m}=2 n-m-1$, then $\lambda_{m+1}<2 n-m-1$.

Theorem 4.6 Let $1 \leq m \leq n$. Let $\lambda \vdash n(n-1)$ with $n-1 \leq \ell(\lambda) \leq n$ and parts $\lambda_{1}=\lambda_{2}=\ldots=$ $\lambda_{m}=2 n-m-1$. Let $\mu \vdash n(n+1)$ with parts $\mu_{1}=\mu_{2}=\ldots=\mu_{m+1}=2 n-m$ and (if $\left.m<n\right)$ $\mu_{j}=\lambda_{j-1}$ for $j=m+2, \ldots, n+1$. Then,

$$
\left\langle a_{\delta_{n+1}}^{2}, s_{\mu}\right\rangle=(-1)^{m}(2 m+1)\left\langle a_{\delta_{n}}^{2}, s_{\lambda}\right\rangle .
$$

Thus, adding the tetris type shape

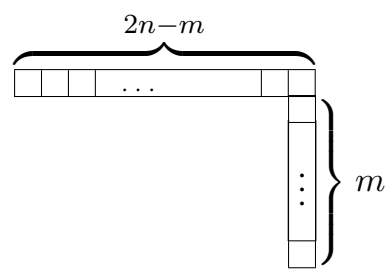

to the top of the diagram of $\lambda$ changes the coefficient by a multiple of $(-1)^{m}(2 m+1)$.

Proof: The proof relies on tricky but elementary linear algebra and reduces to [6, Exercise 7.37(b)] which shows that

$$
\left\langle a_{\delta_{m}}^{2}, s_{\left\langle(m-1)^{m}\right\rangle}\right\rangle=(-1)^{\left(\begin{array}{c}
m \\
2
\end{array}\right)} \cdot 1 \cdot 3 \cdots(2 m-1) .
$$


Note: If $k=1$, Theorem 4.1 fits into the pattern of Theorem 4.6 for $m=0$.

Exercise 7.37(c) of [6] follows as an easy corollary of Theorems 4.6 and 4.1

Corollary 4.7 If $\lambda=\left((n+i-1)^{n-i},(i-1)^{i}\right), 1 \leq i \leq n$, then

$$
\left\langle a_{\delta_{n}}, s_{\lambda}\right\rangle=(-1)^{\frac{1}{2}(n-1)(n-2 i)}[1 \cdot 3 \cdots(2 i-1)] \cdot[1 \cdot 3 \cdots(2(n-i)-1)] .
$$

We state conjecturally a similar combinatorial recursive property.

Conjecture 4.8 Let $1 \leq m \leq n-1$. Let $\lambda \vdash n(n-1)$ with $n-1 \leq \ell(\lambda) \leq n$ and parts $\lambda_{1}=\lambda_{2}=$ $\ldots=\lambda_{m}=2 n-m-2$. Let $\mu \vdash n(n+1)$ with parts $\mu_{1}=2 n-m, \mu_{2}=\ldots=\mu_{m+1}=2 n-m-1$ and (if $m<n) \mu_{j}=\lambda_{j-1}$ for $j=m+2, \ldots, n+1$. Then,

$$
\left\langle a_{\delta_{n+1}}^{2}, s_{\mu}\right\rangle=(-1)^{m}(m+1)\left\langle a_{\delta_{n}}^{2}, s_{\lambda}\right\rangle .
$$

Thus adding the tetris type shape

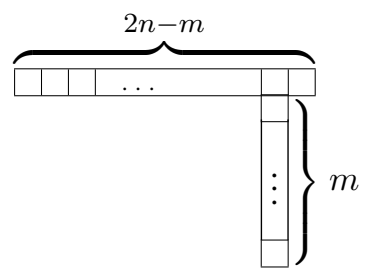

to the top of the diagram of $\lambda$ changes the coefficient by a multiple of $(-1)^{m}(m+1)$.

The statement of the conjecture will be proved if we can show the following.

Let $l=\left(l_{1}, l_{2}, \ldots, l_{m}, l_{m+1}=0\right)$ be a partition of $m$ of length at most $m+1$ (thus, at least one part is zero). Let $\alpha$ be a permutation of $\{1,2, \ldots, m\}$ and let $\beta$ be a permutation of $\{1,2, \ldots, m, m+1\}$. Denote by $\mathcal{C}(l, \alpha)$ the coefficient of

$$
x_{1}^{2 m-3+l_{\alpha(1)}} x_{2}^{2 m-4+l_{\alpha(2)}} \cdots x_{m}^{m-2+l_{\alpha(m)}}=x^{\left\langle(m-2)^{m}\right\rangle+\alpha\left(l^{*}\right)+\delta_{m}}
$$

in $a_{\delta_{m}}^{3}$ (here, $\left.l^{*}=\left(l_{1}, l_{2}, \ldots, l_{m}\right)\right)$ and denote by $\overline{\mathcal{C}}(l, \beta)$ the coefficient of

$$
x_{1}^{2 m+l_{\beta(1)}} x_{2}^{2 m-2+l_{\beta(2)}} x_{3}^{2 m-3+l_{\beta(3)}} \cdots x_{m}^{m+l_{\beta(m)}} x_{m+1}^{m-1+l_{\beta(m+1)}}=x^{\left\langle m,(m-1)^{m}\right\rangle+\beta(l)+\delta_{m+1}}
$$

in $a_{\delta_{m+1}}^{3}$.

We need to show that for each partition $l=\left(l_{1}, l_{2}, \ldots l_{m}\right)$ of $m$ of length at most $m$,

$$
\sum_{\beta \in S_{m+1}} \overline{\mathcal{C}}(l, \beta)=(-1)^{m}(m+1) \sum_{\alpha \in S_{m}} \mathcal{C}(l, \alpha) .
$$

The particular cases of Conjecture 4.8 when $m=1$ or $m=n-1$ can be proved directly. 
Proposition 4.9 (Case $m=1$ of Conjecture 4.8) Let $\lambda=\left(2 n-3, \lambda_{2}, \ldots, \lambda_{n}\right) \vdash n(n-1)$ and $\mu=\left(2 n-1,2 n-2, \lambda_{2}, \ldots, \lambda_{n}\right) \vdash n(n+1)$. Then

$$
\left\langle a_{\delta_{n+1}}^{2}, s_{\mu}\right\rangle=-2\left\langle a_{\delta_{n}}^{2}, s_{\lambda}\right\rangle
$$

Proof: The proof is elementary and relies on a case analysis of the contribution of $\left(x_{1}-x_{2}\right)^{3}$ to the coefficient of $x^{\mu+\delta_{n+1}}$ in $a_{\delta_{n+1}}^{3}$.

Using Proposition 4.9 and Theorem 4.1 we can prove by induction the following observation that Dunne finds remarkable (see [2, Section 6]). Starting with the partition $\lambda$ of Corollary $4.2, \lambda=(2(n-1), 2(n-$ $2), \ldots, 4,2,0)$, if we remove the last box from the $j$ th row of the Young diagram of $\lambda$ and add it to the end of the $(j+k)$ th row, the coefficient changes to $(-1)^{k} \cdot 3 \cdot 2^{k-1}$.

Corollary 4.10 Fix an integer $j$ with $1 \leq j \leq n-1$ and let $k$ be a fixed integer such that $j+1 \leq k \leq n$. If $\nu \vdash n(n-1)$ is given by

$(2(n-1), \ldots, 2(n-j+1), 2(n-j)-1,2(n-j-1), \ldots, 2(n-k+1), 2(n-k)+1,2(n-k-1), \ldots, 2,0)$,

then

$$
\left\langle a_{\delta_{n}}, s_{\nu}\right\rangle=(-1)^{k-j} \cdot 3 \cdot 2^{k-j-1} .
$$

Proposition 4.11 (Case $m=n-1$ of Conjecture 4.8 Let $\lambda=\left\langle(n-1)^{n}\right\rangle \vdash n(n-1)$ and $\mu=$ $\left\langle n+1, n^{n}\right\rangle \vdash n(n+1)$. Then

$$
\left\langle a_{\delta_{n+1}}^{2}, s_{\mu}\right\rangle=(-1)^{n-1} n\left\langle a_{\delta_{n}}^{2}, s_{\lambda}\right\rangle .
$$

Proof: We first introduce some definitions following [6, Chapter 7]. Denote by $f_{\lambda}$ the number of standard Young tableaux (SYT) of shape $\lambda$. Given a Young diagram $\lambda$ and a square $u=(i, j)$ of $\lambda$, let $h(u)$ denote the hook length of $u$, the number of squares directly to the right or directly below $u$, including $u$ itself once. Thus

$$
h(u)=\lambda_{i}+\lambda_{j}^{\prime}-i-j+1 .
$$

We also define the content $c(u)$ of $\lambda$ at $u=(i, j)$ by

$$
c(u)=j-i .
$$

If $\lambda$ is a partition of $t$, the hook-length formula [6, Corollary 7.21.6] gives

$$
f_{\lambda}=\frac{t !}{\prod_{u \in \lambda} h(u)} .
$$

If $\lambda$ is a partition of $n(n-1)$ and $\lambda=\eta+\left\langle(n-2)^{n}\right\rangle$ (thus $\eta$ is a partition of $n$ ), then, by [6, Exercise 7.37.d]

$$
\left\langle a_{\delta_{n}}^{2}, s_{\lambda}\right\rangle=(-1)^{\left(\begin{array}{c}
n \\
2
\end{array}\right)} f_{\eta} \prod_{s \in \eta}(1-2 c(s)) .
$$


As noted in [4],

$$
f_{\lambda}=\sum_{\nu \in \lambda \backslash 1} f_{\nu}
$$

where $\lambda \backslash 1$ is the set of partitions obtained from $\lambda$ by removing a corner. (This formula follows directly from the construction of standard Young tableaux.)

For the partitions $\lambda$ and $\mu$ in the statement of the proposition, we have

$$
\lambda=\left\langle(n-1)^{n}\right\rangle=\left\langle 1^{n}\right\rangle+\left\langle(n-2)^{n}\right\rangle
$$

and

$$
\mu=\left\langle 2,1^{n-1}\right\rangle+\left\langle(n-1)^{n}\right\rangle
$$

Then, by $[26$, we have

$$
\left\langle a_{\delta_{n}}^{2}, s_{\lambda}\right\rangle=(-1)^{\left(\begin{array}{c}
n \\
2
\end{array}\right)} f_{\left\langle 1^{n}\right\rangle} \prod_{s \in\left\langle 1^{n}\right\rangle}(1-2 c(s))=(-1)^{\left(\begin{array}{c}
n \\
2
\end{array}\right)} 1 \cdot 3 \cdot 5 \cdots(2 n-1)
$$

and

$$
\begin{gathered}
\left\langle a_{\delta_{n}}^{2}, s_{\mu}\right\rangle=(-1)^{\left(\begin{array}{c}
n+1 \\
2
\end{array}\right)} f_{\left\langle 2,1^{n-1}\right\rangle} \prod_{s \in\left\langle 2,1^{n-1}\right\rangle}(1-2 c(s))= \\
(-1)^{\left(\begin{array}{c}
n+1 \\
2
\end{array}\right)} \frac{(n+1) !}{(n+1)(n-1) !}(-1) \cdot 1 \cdot 3 \cdot 5 \cdots(2 n-1)=(-1)^{n-1} n\left\langle a_{\delta_{n}}^{2}, s_{\lambda}\right\rangle .
\end{gathered}
$$

Theorem 4.12 Let $\lambda \vdash n(n-1)$ with $\ell(\lambda)=n-1$ and $\lambda_{n-1} \geq n-1$ and let $\mu \vdash n(n+1)$ be given by $\mu=\left(\lambda_{1}+1, \lambda_{2}+1, \ldots, \lambda_{n-1}+1, n, 1\right)$. Then,

$$
\left\langle a_{\delta_{n+1}}^{2}, s_{\mu}\right\rangle=(-1)^{n} 3 n\left\langle a_{\delta_{n}}^{2}, s_{\lambda}\right\rangle .
$$

Thus adding the tetris type shape

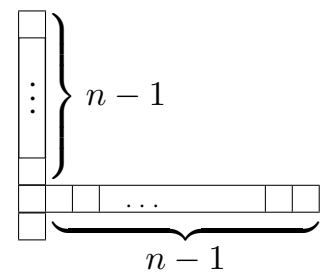

to the left of the diagram of $\lambda$ changes the coefficient by a multiple of $(-1)^{n} 3 n$.

Proof: If $\lambda \vdash n(n-1)$ with $\ell(\lambda)=n-1$ is as in the statement of the theorem, then $\lambda_{n-1}$ equals $n$ or $n-1$.

Case I: $\lambda_{n-1}=n$. Then $\lambda=\left\langle n^{n-1}\right\rangle$ and, after repeatedly applying Theorem 4.6, we have

$$
c_{\lambda}:=\left\langle a_{\delta_{n}}^{2}, s_{\lambda}\right\rangle=(-1)^{\left(\begin{array}{c}
n-1 \\
2
\end{array}\right)} 1 \cdot 3 \cdot 5 \cdots(2 n-3) .
$$


In this case $\mu=\left\langle(n+1)^{n-1}, n, 1\right\rangle$ and the calculation of $c_{\mu}=\left\langle a_{d_{n+1}}, s_{\mu}\right\rangle$ relies on Proposition 4.11

Case II: $\lambda_{n-1}=n-1$. Thus, $\lambda=\left\langle(n-1)^{n-1}\right\rangle+\nu$, where $\nu$ is a partition of $n-1$. The last part of $\nu$ can only be 0 or 1 . If $\nu_{n-1}=1$, then we are in Case I. Therefore, we assume $\nu_{n-1}=0$.

Using Corollary 4.3 , we have

$$
\left\langle a_{\delta_{n}}^{2}, s_{\lambda}\right\rangle=\left\langle a_{\delta_{n-1}}^{2}, s_{\lambda /\left\langle 2^{n-1}\right\rangle}\right\rangle
$$

Since $\lambda /\left\langle 2^{n-1}\right\rangle=\left\langle(n-3)^{n-1}\right\rangle+\nu$ is a partition of $(n-1)(n-2)$, we can use 26) to obtain

$$
\left\langle a_{\delta_{n}}^{2}, s_{\lambda}\right\rangle=(-1)^{\left(\begin{array}{c}
n-1 \\
2
\end{array}\right)} f_{\nu} \prod_{s \in \nu}(1-2 c(s)) .
$$

We can also use 25] to write

$$
\left\langle a_{\delta_{n}}^{2}, s_{\lambda}\right\rangle=(-1)^{\left(\begin{array}{c}
n-1 \\
2
\end{array}\right)} \frac{(n-1) !}{\prod_{u \in \nu} h(u)} \prod_{s \in \nu}(1-2 c(s)) .
$$

Now let us consider $\mu=\left(n+\nu_{1}, n+\nu_{2}, \ldots, n+\nu_{n-1}, n, 1\right)$. We have

$$
x^{\mu+\delta_{n+1}}=x_{1}^{2 n+\nu_{1}} x_{2}^{2 n+\nu_{2}-1} \cdots x_{i}^{2 n-\nu_{i}-i+1} \cdots x_{n-2}^{n+3+\nu_{n-2}} x_{n-1}^{n+2+\nu_{n-1}} x_{n}^{n+1} x_{n+1} .
$$

We write $a_{\delta_{n+1}}^{3}$ as

$$
a_{\delta_{n+1}}^{3}=a_{\delta_{n}}^{3} \prod_{i=1}^{n}\left(x_{i}-x_{n+1}\right)^{3}
$$

For each $i=1,2, \ldots, n$, the product $\prod_{i=1}^{n}\left(x_{i}-x_{n+1}\right)^{3}$ contributes

$$
-3 x_{i}^{2} x_{n+1} x_{1}^{3} x_{2}^{3} \cdots x_{i-1}^{3} x_{i+1}^{3} \cdots x_{n}^{3}
$$

and $a_{\delta_{n}}^{3}$ contributes

$$
x_{1}^{2 n+\nu_{1}-3} x_{2}^{2 n+\nu_{2}-4} x_{3}^{2 n+\nu_{3}-5} \cdots x_{i-1}^{2 n+\nu_{i-1}-i-1} x_{i}^{2 n-\nu_{i}-i-1} x_{i+1}^{2 n+\nu_{i+1}-i-3} \cdots x_{n-1}^{n+\nu_{n-1}-1} x_{n}^{n-2}
$$

with multiplicity $c_{i}$ to forming $x^{\mu+\delta_{n+1}}$.

If $\nu_{i-1}=\nu_{i}$, then $c_{i}=0$. Moreover, $c_{n}=0$ since we assumed that $\nu_{n-1}=0$.

For each $i=1,2, \ldots, n$, such that $\nu_{i-1}>\nu_{i}, c_{i}=\left\langle a_{\delta_{n}}^{2}, s_{\eta_{i}}\right\rangle$, where $\eta_{i}=\left(n+\nu_{1}-2, n+\nu_{2}-2, n+\nu_{3}-2, \ldots, n+\nu_{i-1}-2, n+\nu_{i}-1, n+\nu_{i+1}-2, \ldots, n+\nu_{n-1}-2, n-2\right)$.

Thus

$$
\eta_{i}=\left\langle(n-2)^{n}\right\rangle+\tilde{\nu}_{i}
$$


where $\tilde{\nu}_{i}$ is the partition of $n$ obtained from $\nu$ by adding a box at the end of the $i$ th row, i.e.,

$$
\tilde{\nu}_{i}=\left(\nu_{1}, \nu_{2}, \ldots, \nu_{i-1}, \nu_{i}+1, \nu_{i+1}, \ldots, \nu_{n-1}\right) .
$$

To find $c_{i}$ we can now use 26 . We have

$$
c_{i}=(-1)^{\left(\begin{array}{l}
n \\
2
\end{array}\right)} f_{\tilde{\nu}_{i}} \prod_{s \in \tilde{\nu}_{i}}(1-2 c(s))
$$

or, using [25],

$$
c_{i}=(-1)^{\left(\begin{array}{l}
n \\
2
\end{array}\right)} \frac{n !}{\prod_{u \in \tilde{\nu}_{i}} h(u)} \prod_{s \in \tilde{\nu}_{i}}(1-2 c(s)) .
$$

Now let us compare $\prod_{s \in \nu}(1-2 c(s))$ and $\prod_{s \in \tilde{\nu}_{i}}(1-2 c(s))$. We have

$$
\prod_{s \in \tilde{\nu}_{i}}(1-2 c(s))=\left(1-2 c\left(i, \nu_{i}+1\right)\right) \prod_{s \in \nu}(1-2 c(s))=\left(2 i-2 \nu_{i}-1\right) \prod_{s \in \nu}(1-2 c(s)) .
$$

Thus, using (34) and 39), in order to prove the theorem, we need to show that

$$
n f_{\nu}=\sum_{\substack{i=1 \\ \nu_{i}-1>\nu_{i}}}^{n} f_{\tilde{\nu}_{i}}\left(2 i-2 \nu_{i}-1\right)
$$

Note that the terms for $i=1$ and $i=\ell(\nu)+1$ are always included in the sum.

The Statement (42) can be proved by induction using the commutativity of the operations "removal of one box" and "addition of one box". It also follows from [5].

\section{Open problems}

1. Ideally, one would be able to find other rules involving different tetris type shapes.

2. In [3], the authors define admissible partitions and conjecture that they determine the Schur functions with non-zero coefficients in the decomposition of the $(2 k)$ th power of the Vandermonde determinant. However, the conjecture fails and counterexamples have been provided by [7]. It remains an open problem to find a non-vanishing criterion for these coefficients.

\section{References}

[1] A. Boussicault, J-G Luque, C. Tollu, Hyperdeterminantal computation for the Laughlin wavefunction, J. Phys. A: Math. Theor. 42 (2009) 145301

[2] G. Dunne Slater Decomposition of Laughlin States, Int. J. Mod. Phys. B 7 (1993), 4783-4813 
[3] P. Di Francesco, M. Gaudin, C. Itzykson, and F. Lesage, Laughlin's wave functions, Coulomb gases and expansions of the discriminant, Int. J. Mod. Phys. A 9 (1994), 4237-4351

[4] G.-H. Han, Hook lengths and shifted partitions, arXiv:0807.1801v1 [math.CO]

[5] M. Konvalinka, The weighted hook-length formula II: Complementary formulas, arXiv:1006.1865v1 [math.CO]

[6] R. P. Stanley, Enumerative Combinatorics, Volume 2, Cambridge Studies in Advanced Mathematics 62, Cambridge University Press, 1999.

[7] T Scharff, J-Y Thibon and B G Wybourne, Powers of the Vandermonde determinant and the quantum Hall effect, J. Phys. A: Math. Gen. 27 4211-4219, 1994. 